\title{
Cholinergic Modulation of Visual Attention and Working Memory: Dissociable Effects of Basal Forebrain 192-IgG-saporin Lesions and Intraprefrontal Infusions of Scopolamine
}

\author{
Yogita Chudasama, ${ }^{1,2,3}$ Jeffrey W. Dalley, ${ }^{1}$ Falgyni Nathwani, ${ }^{1}$ Pascale Bouger, ${ }^{1}$ \\ and Trevor W. Robbins ${ }^{1}$ \\ ${ }^{1}$ Cambridge University, Department of Experimental Psychology, Cambridge CB2 3EB, UK
}

Two experiments examined the effects of reductions in cortical cholinergic function on performance of a novel task that allowed for the simultaneous assessment of attention to a visual stimulus and memory for that stimulus over a variable delay within the same test session. In the first experiment, infusions of the muscarinic receptor antagonist scopolamine into the medial prefrontal cortex (mPFC) produced many omissions but did not impair rats' ability to correctly detect a brief visual stimulus. However, these animals were highly impaired in remembering the location of that stimulus following a delay period, although in a delay-independent manner. In the second experiment, another group of animals with selective 192 lgG-saporin lesions of the nucleus basalis magnocellularis (nBM) were not impaired under conditions of low-attentional demand. However, when the stimulus duration was reduced, a significant memory impairment was observed, but similar to the results of the first experiment, the nBM-lesioned animals were not impaired in attentional accuracy, although aspects of attention were compromised (e.g., omissions). These findings demonstrate that (1) cortical cholinergic depletion produces dissociable deficits in attention and memory, depending on the task demands, (2) delay-independent mnemonic deficits produced by scopolamine are probably due to impairments other than simple inattention, and (3) working memory deficits are not simply dependent on attentional difficulties per se. Together, these findings implicate the nBM cortical cholinergic system in both attentional and mnemonic processing.

\begin{abstract}
There has been a long standing interest in the hypothesis that cortical cholinergic dysfunction underlies the cognitive impairments associated with normal aging and dementia (Drachman and Leavitt 1974; Bartus et al. 1982; 1985; Weingartner 1985; Broks et al. 1988; Sahakian et al. 1990; Dunnett and Barth 1991; Robbins et al. 1997; Wenk 1997; Barense et al. 2002). The magnocellular cells of the nucleus basalis of Meynert are significantly deficient in patients with Alzheimer's disease (Whitehouse et al. 1982; Candy et al. 1983), and cholinergic markers in the cortical target fields of basal forebrain neurons are also reduced in these individuals (Davies and Maloney 1976; Perry et al. 1977), which appears to correlate with the degree of dementia (Perry et al. 1978).
\end{abstract}

In normal human volunteers, it is well established that scopolamine, a muscarinic receptor antagonist, impairs the acquisition of new information and disrupts the process of memory consolidation (Drachman and Leavitt 1974; Petersen 1977; Jones et al. 1979; Broks et al. 1988) as well as impairing continuous performance in a task that challenges sustained attentional mechanisms (Colquhoun 1962; Wesnes and Warburton 1983, 1984; Broks et al. 1988). These findings clearly implicate the cholinergic system in both attentional and memory processes, but the precise relationship between these two functions is far from resolved.

In rodents, most tests of working memory depend on defining delay-dependent effects in generic-delayed response tasks

\footnotetext{
2Present addess: Laboratory of Neuropsychology, National Institute of Mental Health, Bethesda, MD 20892, USA.

${ }^{3}$ Corresponding author.

E-MAIL Yogita@In.nimh.nih.gov; FAX (301) 402-0046.

Article and publication are at http://www.learnmem.org/cgi/doi/10.1101/ Im.70904.
}

such as delayed alternation and delayed nonmatching to position. Apparent delay-dependent effects can, however, arise artifactually from scaling constrained by ceiling effects, and do not always adequately assess the contribution of other processes such as stimulus control, attention, and response selection. Hence, behavioral effects of scopolamine infusions into the rat medial prefrontal cortex (mPFC) have been inconclusive. Whereas some have argued that scopolamine produces a specific workingmemory deficit (Granon et al. 1995; Ragozzino et al. 1998), others have shown that scopolamine or $\mathrm{nBM}$ cholinergic lesions produce delay-independent deficits, suggesting, therefore, that the deficit was not primarily mnemonic and more likely a reflection of poor attentional responding (Dunnett et al. 1989, 1991; Robbins et al. 1989; Everitt and Robbins 1997).

This notion has been substantiated recently by the introduction of the immunotoxin 192 IgG-saporin, which has a greater specificity for cholinergic cells, and which produces deficits that are mainly attentional in nature (McGaughy et al. 1996, 2002; Baxter and Chiba 1999; Sarter and Bruno 1999; Robbins 2002) rather than mnemonic (Torres et al. 1994; Wenk et al. 1994; Baxter et al. 1995; Chappell et al. 1998). Most recently, in the 5-choice serial reaction time task (Carli et al. 1983; Robbins 2002), a rat analog of the continuous performance test (Rosvold et al. 1956), intrabasalis infusions of high or low doses of 192 IgG-saporin produced different degrees of damage that correlated with the degree of accuracy deficit (McGaughy et al. 2002). Furthermore, the accuracy deficit was significantly correlated with a reduction in cortical acetylcholine (ACh) efflux in rats with extensive lesions only (McGaughy et al. 2002).

Nevertheless, the conclusion that the cortical cholinergic system subserves attentional rather than mnemonic functions fails to adequately characterize the role of these neurons in cog- 
nitive function (see also Dunnett et al. 1991; Baxter and Chiba 1999). The possibility still remains that the basal forebrain mediates both attentional and memory processes, and the direct role of acetylcholine may be to distribute attentional capacity in tasks that require effortful processing such as holding a stimulus on-line. However, it is difficult to disentangle the attentional component from memory during a delayed-response paradigm and equally difficult to explore the working memory component incorporated within the 5CSRTT. To this end, we have developed a novel paradigm using the 9-hole box (Robbins 2002) in order to explore the rats' ability to detect brief visual targets, and then to retain their locations over varying delays. In this combined attention-memory (CAM) task, rats attained $>70 \%$ accuracy at $0.7-$ $1.0-\mathrm{sec}$ stimulus duration for the attentional component (chance $=20 \%$ ) and a delay-dependent gradient from 0-16 sec of $\sim 70 \%-50 \%$ (chance $=50 \%$ ) for the memory component. In experiment 1 , trained rats were implanted with guide cannulae in the $\mathrm{mPFC}$, and then received three doses of intra-mPFC scopolamine infusions (vehicle, 3 or $10 \mu \mathrm{g}$ ) in a counterbalanced order. In experiment 2 , another cohort of trained rats received 192 IgGsaporin lesions of the nBM to examine the effects of selective cortical cholinergic depletion on attention and memory. Following postoperative recovery, the rats were tested for $8 \mathrm{~d}$ on the baseline schedule of the task. Following this, in one session, the stimulus duration was reduced from 1.0 to $0.25 \mathrm{sec}$ to enhance attentional encoding demands.

\section{RESULTS}

\section{Experiment 1: Intramedial Prefrontal Cortex Infusions of Scopolamine}

\section{Histological Analysis}

Figure 1 shows a schematic representation of the position of the cannula tips within the mPFC. The cannula tips were located mainly in the anterior cingulate cortex $(\mathrm{Cg} 1)$ and prelimbic $(\mathrm{PrL})$ region of the mPFC. Cytoarchitectonic borders and nomenclature were taken from Paxinos and Watson (1997). One animal died during surgery, therefore, a total of 13 rats were used for this study.

\section{Behavioral Results}

\section{Postoperative Baseline (Five Sessions)}

Following $7 \mathrm{~d}$ of post operative recovery, the animals were reintroduced to the standard baseline schedule for $5 \mathrm{~d}$. The group of

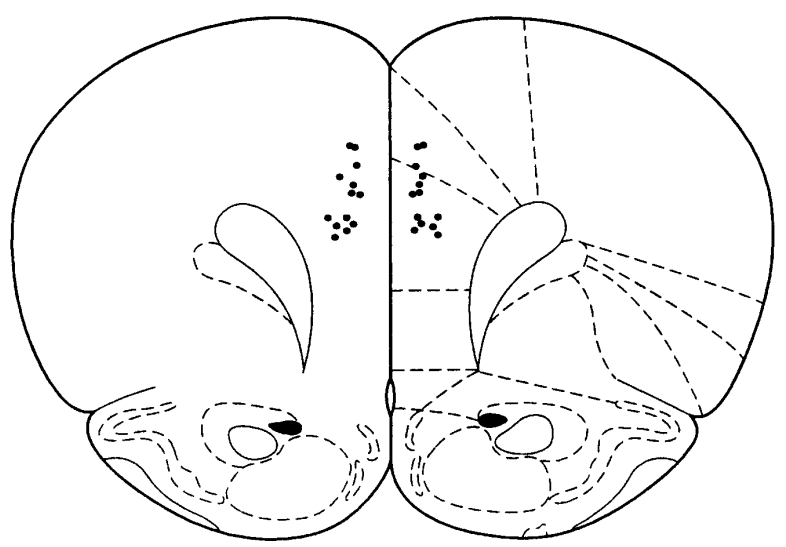

Figure 1 Schematic diagram showing location of cannula tips in the medial prefrontal cortex (mPFC). Atlas plates adapted from Paxinos and Watson (1997). Section +3.2 mm from bregma. animals showed accurate performance on attentional accuracy across all sessions $[\mathrm{F}(4,48)=0.17, P>0.05$; mean of five sessions, $77.1 \%( \pm 2.2 \mathrm{SEM})]$ that was associated with a low number of target omissions $[\mathrm{F}(4,48)=2.09, P>0.05$; mean of five sessions, $6.9 \%( \pm 1.1)]$. There was no differential effect of session on the latency to respond to the target $[P>0.05$; mean in csec, 73.18]. The number of premature responses declined over sessions $[\mathrm{F}(4$, $48)=2.45, P=0.059$; mean $( \pm \mathrm{SEM})$ for session $1,92(13)$, mean for session 5,85 (15)]. There was no significant change over sessions in the number of perseverative nose pokes made in the holes during the delay $[\mathrm{F}(4,48)=1.35, P>0.05]$. During the memory phase, these animals showed a delay-dependent decline in choice accuracy $[\mathrm{F}(3,36)=41.9, P<0.01$; means $( \pm \mathrm{SEM})$ : $0 \mathrm{~s}$ $72.1 \%$ (1.8), 4s- 69.6\% (1.6), 8s- 64.8\% (1.3), 16s- 53.7\% (1.3)], but there was no main effect of session $[\mathrm{F}(4,48)=1.31, P>0.05]$. Latencies to respond to the choice stimuli or collect food reward were also unaffected $(P>0.05)$.

\section{Effect of Scopolamine Infusions Into the Medial Prefrontal Cortex}

Figure $2 \mathrm{~A}$ shows that there was no significant effect of dose on attentional accuracy $[\mathrm{F}(2,24)=1.53, P<0.05]$. However, these animals made significantly more omissions at the high dose $[\mathrm{F}(2$, $24)=9.42, P<0.01$; see Fig. $2 \mathrm{~B}]$ and were also slower to respond to the target $[\mathrm{F}(2,24)=7.55, P<0.01$; means $( \pm \mathrm{SEM})$ in csec, veh- 67.2 (2.93), $3 \mu \mathrm{g}$ dose- 67.2 (2.89), $10 \mu \mathrm{g}$ dose- 77.5 (2.85)]. ANOVA showed a dose-dependent effect of scopolamine on the number of premature responses $[\mathrm{F}(2,24)=5.93, P<0.01]$ that was characterized by an increase in the number of responses made at the low dose and a reduction in responses made at the high dose [means ( \pm SEM), veh- 113 (21.2), $3 \mu$ g dose- 137 (21.5), $10 \mu \mathrm{g}$ dose- 94 (20.8)]. The number of perseverative nose pokes made during the delay also significantly reduced at the high dose $[\mathrm{F}(2,24)=4.12, P<0.01$; means $( \pm$ SEM $)$, veh- 98 (18.9), $3 \mu \mathrm{g}$ dose- 93 (18.0), $10 \mu \mathrm{g}$ dose- 67 (16.9)].

During the memory phase of the task, overall, choice accuracy systematically declined as a function of delay $[\mathrm{F}(3$, $36)=11.71, P<0.001]$. The high dose of scopolamine produced a delay-independent reduction in choice accuracy across all delays $[\mathrm{F}(2,24)=13.31, P<0.001$; see Fig. $2 \mathrm{C}]$. There was no dose $\mathrm{x}$ delay interaction $[\mathrm{F}(6,72)=0.45, P>0.05]$. In addition, the high dose of scopolamine made all animals slower to respond correctly to the choice stimulus $[\mathrm{F}(2,24)=5.60, P<0.01$; means ( \pm SEM) in csec, veh- 116 (4.70), $3 \mu \mathrm{g}$ dose- 111 (3.22), $10 \mu \mathrm{g}$ dose- $123(3.79)]$ and lengthened magazine latencies $[\mathrm{F}(2$, $24)=6.71, P<0.01$; means $( \pm$ SEM) in csec, veh- $143(8.86), 3 \mu \mathrm{g}$ dose- 143 (8.20), $10 \mu \mathrm{g}$ dose- 220 (36.25)].

Thus, although a high dose of scopolamine infused into the mPFC did not impair the animals' ability to correctly detect the target in the attentional phase, these animals were unable to accurately respond to the matching target stimulus during the choice (memory) phase of the task, even at the short delay. Speed of responding was also significantly impaired.

\section{Experiment 2: Effects of nBM-Saporin Lesions on Performance of the CAM Task}

\section{Histological Analysis}

Representative photomicrographs of the saporin lesion in the basal forebrain are shown in Figure 3. Examination of the sections revealed a substantial reduction in the number of ChATimmunoreactive (IR) neurons throughout the full extent of the Ch4 region of the basal forebrain in 192-IgG-lesioned-animals. The lesion was assessed in a semiquantitative manner by counting ChAT-IR neurons in the Ch4 region (Mesulam et al. 1983a,b) 

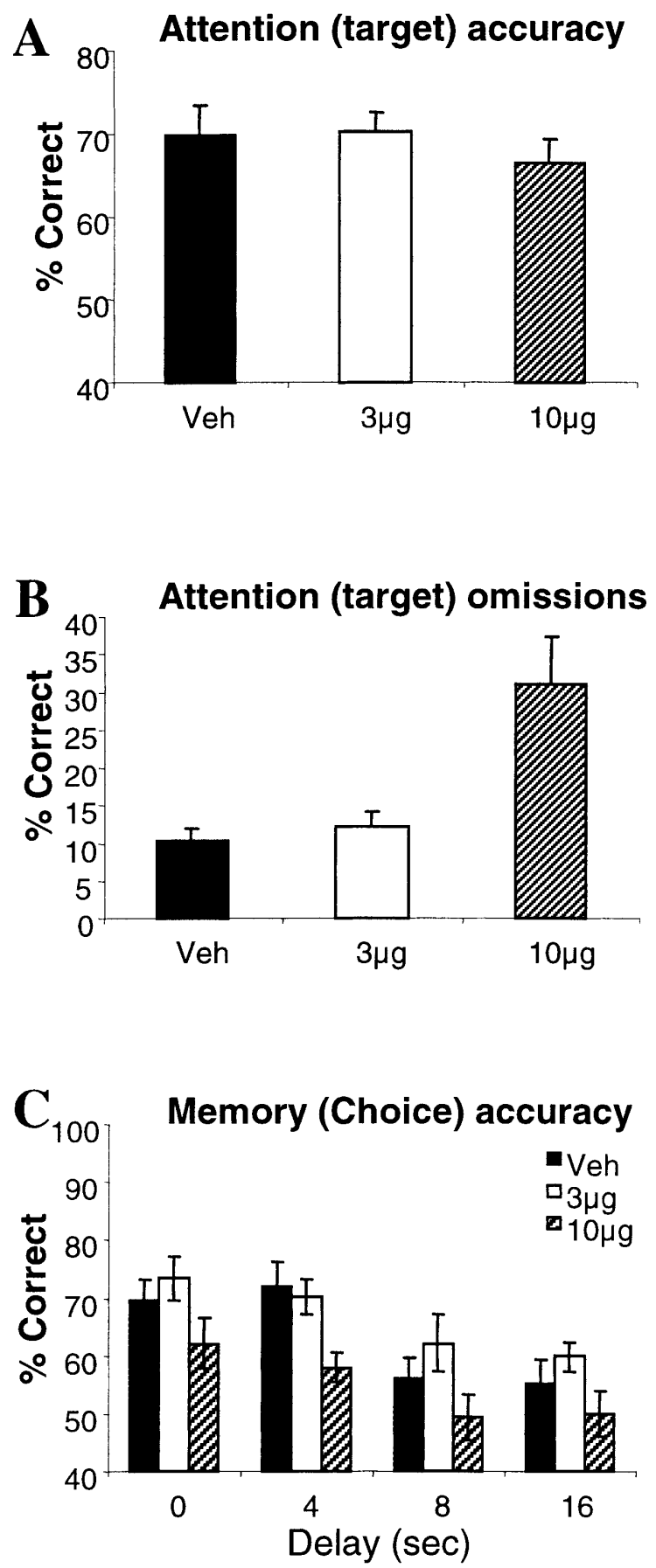

Figure 2 Mean ( \pm SEM) performance of animals with intra-mPFC infusions of vehicle, 3 and $10 \mu \mathrm{g}$ scopolamine on attentional (target) accuracy $(A)$, target omissions $(B)$, and memory for the target during the choice phase (C).

of a representative section of the basal forebrain (roughly -0.6 $\mathrm{mm}$ posterior to bregma). This analysis revealed a $>85 \%$ reduction in ChAT-IR neurons [mean $( \pm$ SEM) number of ChAT-IR cells in the left and right hemispheres, respectively; shams, left 44 (7), right 52 (7), lesion, left 3 (1), right 6 (1)], which was comparable in magnitude with the low-dose saporin lesions made in our earlier study (McGaughy et al. 2002). There was no significant indication that ChAT-IR neurons in adjacent nuclei of the basal forebrain, including the horizontal and vertical limbs of diagonal band and septum were decreased in number, and there was no evidence of gross nonselective neuronal damage such as cavitation. In addition, parvalbumin-IR neurons within the basal forebrain were unaffected by the 192-IgG-saporin infusions (see also, McGaughy et al. 2002).

\section{Behavioural Results}

\section{Postoperative Baseline (Eight Sessions)}

Over the eight postoperative baseline sessions, all animals improved attentional accuracy $[\mathrm{F}(7,77)=5.80, P<0.01]$, but there was no effect of group on this measure $[\mathrm{F}(1,11)=2.48, P>0.05$; see Fig. 4A]. Figure 4B shows that the nBM-lesioned animals made more target omissions across all sessions [means $( \pm$ SEM), shams-7.4\% (1.3), nBM-13.6\% (4.7)], but there was no significant effect of lesion on this measure $[\mathrm{F}(1,11)=2.25, P>0.05]$ or on target response latency $[\mathrm{F}(1,11)=2.88, P>0.05$; means $( \pm$ SEM) in csec for session 8 , shams-64.9 (4.62), nBM-77.2 (9.33)]. The number of premature responses $[\mathrm{F}(7,77)=2.42$, $P<0.05]$ and perseverative nose pokes $[\mathrm{F}(7,77)=2.82, P<0.01]$ remained stable over sessions and was unaffected by lesion group $[\mathrm{F}<1]$. Choice performance accuracy in the memory phase improved over session $[\mathrm{F}(7,77)=3.80, P<0.001$; see Fig. $4 \mathrm{C}]$. ANOVA also showed a delay-dependent effect $[\mathrm{F}(3,33)=12.5$, $P<0.001]$, but there was no differential effect of lesion $[\mathrm{F}(1$, $11)=1.08, P>0.05]$. Similarly, there was no effect of lesion group on choice response or magazine latency $(P>0.05)$.

\section{Reduced Stimulus Duration (25 csec)}

Reducing the stimulus duration to $25 \mathrm{csec}$ led to a reduction in attentional accuracy $[\mathrm{F}(1,11)=149.16, P<0.001]$, but there was no significant difference between the sham and lesion groups $[\mathrm{F}(1,11)=0.99, P>0.05$; see Fig. $5 \mathrm{~A}]$. Similarly, both groups of animals showed an increase in the number of target omissions $[(\mathrm{F} 1,11)=13.81, P<0.01]$. The nBM-lesioned group made fewer premature responses during the standard stimulus duration, but made more premature responses when the stimulus duration was reduced, as revealed by a lesion $\mathrm{x}$ stimulus duration interaction $[\mathrm{F}(1,11)=4.45, P<0.05]$. In contrast, the number of perseverative nose pokes made during the delay declined at the reduced stimulus duration $[\mathrm{F}(1,11)=51.96, P<0.001]$, although the nBM-lesioned group was not significantly impaired in either condition $[\mathrm{F}(1,11)=0.01, P<0.05]$.

Figure $5 \mathrm{~B}$ shows percent accuracy scores across all delays during the memory phase of the task. Although there was a delay-dependent reduction in choice response accuracy, this was not quite significant overall $[\mathrm{F}(3,33)=0.06, P>0.05]$. Nonetheless, nBM-lesioned animals were significantly impaired in choice accuracy during the memory phase compared with sham controls, particularly at the long delays $[\mathrm{F}(1,10)=12.01, P<0.01]$. Speed of responding was not affected during this testing schedule $(\mathrm{F}<1)$.

In summary, saporin lesions of the nBM did not impair attention to the target or memory for that target under baseline conditions. When the stimulus duration was reduced, although the nBM group had many errors of omission, they showed no impairment in target detection relative to sham controls. Nonetheless, rats with nBM lesions were unable to remember the stimulus that they had attended to during the memory phase of the task.

\section{DISCUSSION}

Using a novel procedure (CAM task) that allows the separate assessment of attentional and working memory capacities within the same test session, we have demonstrated that reducing cortical cholinergic transmission produces dissociable deficits in at- 

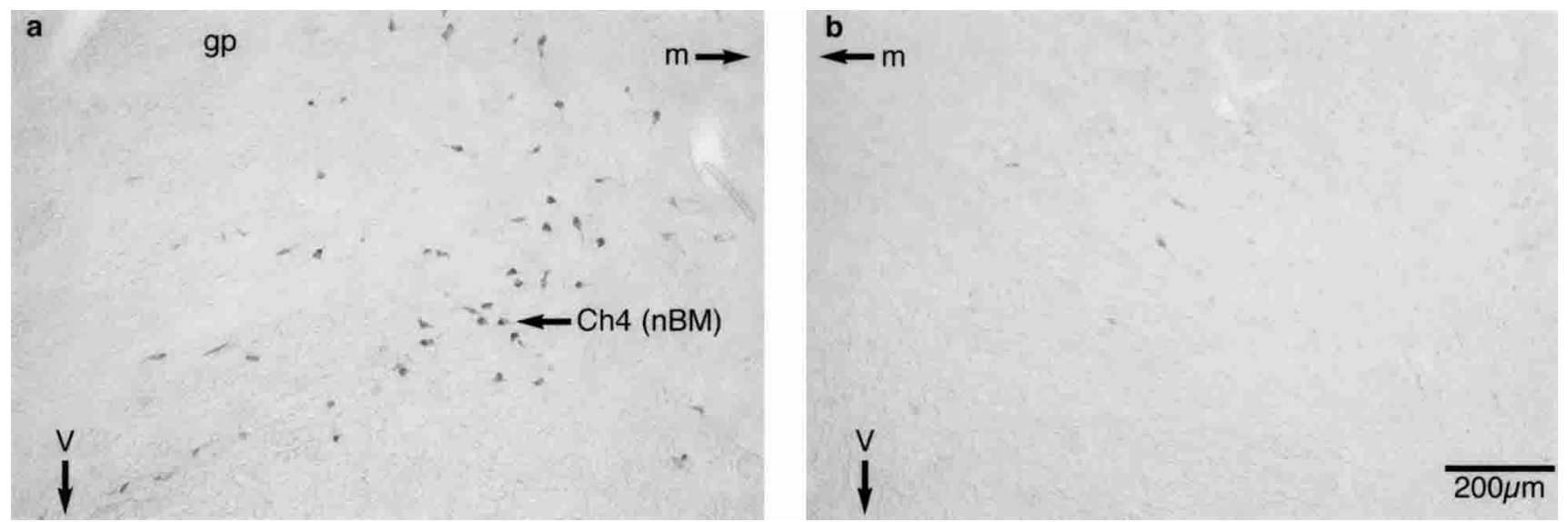

Figure 3 Representative photomicrographs of sections of the basal forebrain showing ChAT-IR neurons in the Ch4 region of sham (a, left) and 192 IgG-saporin lesioned $(b$, right) rats. It can be seen that the magnocellular ChAT-IR neurons of the Ch4 cell group (nucleus basalis magnocellularis; $\mathrm{nBM}$ ) are greatly reduced in number after intrabasalis infusions of $192 \mathrm{lgG}$-saporin. (v) Ventral; (m) medial; (gp) globus pallidus.

tention and memory. Specifically, a high dose of intra-mPFC scopolamine produced a marked decrement in memory for the target stimuli across all delays, although there was no obvious attentional impairment in terms of accuracy. When the cortical cholinergic projections were depleted selectively by intrabasalis infusions of 192 IgG-saporin, attentional and mnemonic performance was relatively unaffected. However, increasing the attentional load by reducing the target duration to $0.25 \mathrm{sec}$ impaired memory performance. These data implicate cortical acetylcholine in working memory function, particularly when the attentional demands are high.

The pronounced memory deficit observed following intramPFC scopolamine infusions is consistent with earlier reports showing delay-independent deficits following scopolamine treatment in operant-delayed alternation paradigms (Dunnett et al. 1990, 1991; Everitt and Robbins 1997). It is tempting to suggest, therefore, that these animals failed to attend or encode the target stimulus, resulting in gross memory impairments even at the short delays; an interpretation consistent with nonspecific memory deficits (Dunnett and Barth 1991). However, the present data fail to support this account, on the grounds that subjects in the present study had already attended to and responded to the targets that they were then required to remember. These data are thus important in demonstrating a deficit in working memory that is not simply dependent on an attentional deficit per se. Nevertheless, intra-mPFC scopolamine at the high dose did produce evidence of a performance deficit in the attention phase. Although attentional accuracy was not impaired, the rats exhibited increased omissions and were slow both to respond and to collect food, suggesting possible deficits in arousal and motivation. It seems unlikely that the effect of scopolamine on working memory can be attributed simply to impaired motivation, as the accuracy deficit in the memory phase was not paralleled by an accuracy deficit in the attentional phase. There was less evidence of impairment in other aspects of executive control of responding, as neither premature nor perseverative responding was significantly increased.

A previous study (Robbins et al. 1998) also reported effects of intra-mPFC scopolamine on the 5CSRTT. These authors found that a $10-\mu \mathrm{g}$ dose of scopolamine significantly reduced attentional accuracy as well as impairing other aspects of performance. However, these rats were trained at a target duration of $0.5 \mathrm{sec}$ rather than the $0.7 \mathrm{sec}$ of the present experiment, which had been used to ensure good behavioral performance at the attentional phase. Overall, therefore, the data are consistent with both at- tentional and working memory deficits of the intra-mPFC infusions, depending on the precise task requirements.

The integrity of the cortical cholinergic system in memory function was further supported by the impairments observed in animals with selective saporin-induced nBM lesions, although, as in the case of intracortical scopolamine, their deficits were also not restricted to memory. A working memory deficit in the saporin nBM-lesioned rats emerged under certain test conditions. Specifically, when the target duration in the attentional phase was reduced to $0.25 \mathrm{sec}$, although both the sham and nBMlesioned trained rats performed well above chance level. However, the nBM group was significantly impaired when a delay was introduced, especially at the longest delays (Fig. 4B).

The evidence of mild nonsignificant attentional deficits, for example, the increase in omissions (Fig. 2B) is not uncommon in animals at this dose of saporin. In a recent study by Risbrough et al. (2002), nBM-saporin-induced lesions using a dose similar to the one used in the present study, also reported increased target omissions under demanding attentional constraints of reduced target duration and intensity, although response accuracy was not significantly affected. In fact, we deliberately focused on this low dose of saporin so as to ensure that profound attentional impairments were minimized while measuring working memory performance. There was also some evidence of impaired inhibitory response control, which emerged at the short stimulus duration with increased premature responding in the nBM trained rats. Such deficits, as well as attentional impairments, are readily discernible at higher doses of saporin (McGaughy et al. 2002), which damage neurons of the horizontal diagonal band of Broca (HDB) in addition to nBM neurons. Notably, both cell groups are known to innervate the mPFC (Saper 1984; Luiten et al. 1987), and this may be relevant to the severity of the attentional impairments observed following intrabasalis infusions of 192 IgGsaporin. The low dose of saporin used in the present study preferentially destroyed nBM, but spared HDB cholinergic neurons (Mesulam et al. 1983a,b). Therefore, it is possible that greater effects may have been observed following a larger lesion that included both the nBM and HDB. Nevertheless, the finding that local infusions of scopolamine into the PFC produced deficits similar to the $\mathrm{nBM}$ lesions suggests that selective cholinergic depletion in the $\mathrm{nBM}$ is sufficient to affect muscarinic transmission in the mPFC

Thus, animals with restricted nBM saporin lesions appear not to be impaired in attentional accuracy under standard conditions, and accordingly, produce no change in extracellular ACh 

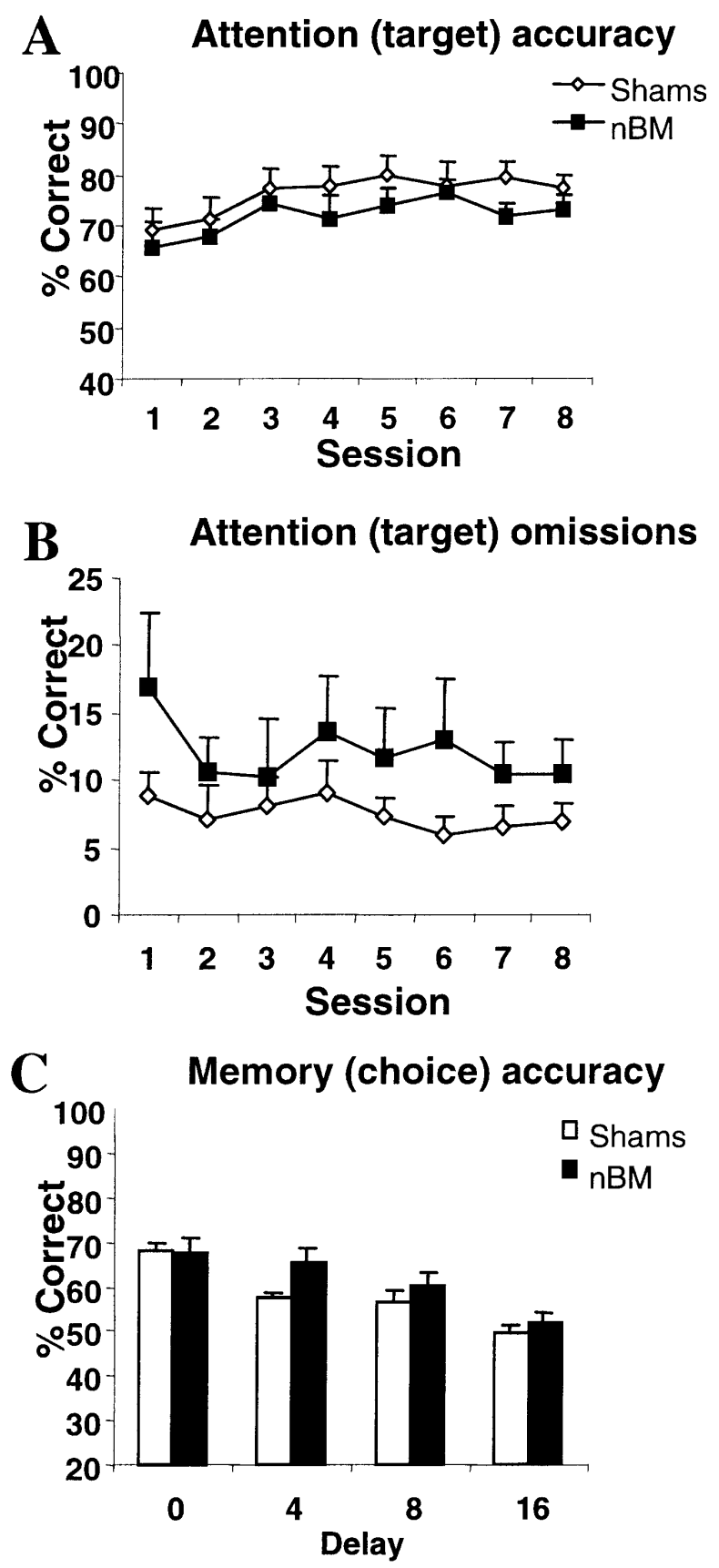

Figure 4 Mean $( \pm$ SEM) performance of Sham controls and nBM lesioned animals on postoperative baseline testing for $8 \mathrm{~d}$ on attentional (target) accuracy $(A)$, target omissions $(B)$, and memory for the target during the choice phase (C).

during performance of the 5CSRTT (see McGaughy et al. 2002). One possibility is that the basal forebrain cortical cholinergic system may be subject to functional compensation following selective cell loss in the $\mathrm{nBM}$, for example, up-regulation of nicotinic and muscarinic receptors in the cortex (e.g., Rossner et al. 1995; Schleibs et al. 1996), and so we cannot be sure how cholinoreceptor density after the lesion may contribute to the benign effects of the lesion under low-attentional demands. Nonetheless, behavioral changes are likely to be more apparent soon, rather than late after the lesion, and this time dependency may be relevant for functional receptor changes, including resprout- ing of surviving neurons and increased synthesis that may emerge with time. Furthermore, deficits induced by cholinergic depletion appear to be dependent on task requirements (Waite et al. 1999; Himmelheber et al. 2001; McGaughy et al. 2002). Similarly, the nBM-lesioned rats in the present study only showed a memory impairment when the attentional demands were high, but not under the standard baseline condition, suggesting that damage to the basal forebrain cholinergic neurons may be critical in optimizing performance in response to specific behavioral challenges (Dunnett et al. 1991; Baxter and Chiba 1999), and this notion is compatible with the role of the cholinergic system in processes of cortical arousal (Richardson and DeLong 1988; Everitt and Robbins 1997).

These observations provide further insight into the modulatory effect of the cortical cholinergic system in attention and memory. Current views hold that selective lesions of the basal forebrain cholinergic neurons produce disruptions in specific forms of attentional processing (Robbins et al. 1989; Chiba et al. 1995, 1999; McGaughy et al. 1996; Stoehr et al. 1997; Turchi and Sarter 1997; Waite et al. 1999) rather than specific mnemonic functions (Etherington et al. 1987; Torres et al. 1994; Wenk 1997; Chappell et al. 1998). However, few, if any, studies have directly compared effects of attention and memory using 192 IgGsaporin in the same animals within the same behavioral task. Deficits produced by less-selective methods for damaging cortical cholinergic projections were taken to support a role for ACh in the cortical plasticity that underlies learning and memory (Dunnett et al. 1991; Everitt and Robbins 1997). There is considerable evidence that central cholinergic neurons do have an important role in mnemonic processing (Hagan and Morris 1988; Sahakian 1988; Fibiger 1991). Interestingly, however, excitotoxic lesions of the mPFC produce working memory deficits (Dunnett et al. 1990; Kesner 2000; Dias and Aggleton 2002), particularly when the task requires effortful processing (Granon et al. 1994), and this is consistent with the finding that $\mathrm{mPFC}$ lesions also produce profound attentional impairments (Muir et al. 1996; Passetti et al. 2002). Together, these data indicate that ACh in the frontal cortex plays a key role in maintaining memory over time as well as serving to optimize attentional processing. Selective excitotoxic lesions of the dorsal prefrontal cortex produce attentional impairments in the 5CSRTT, whereas the more ventral prelimbic cortex appears to be sensitive to performance requiring working memory, including holding information on-line and inhibition of interference from competing traces. (Delatour and Gisquet-Verrier 2000; Passetti et al. 2002; Chudasama et al. 2003). Therefore, attentional and memory functions of ACh may be attributable to cholinergic projections to selective frontal areas. This hypothesis needs to be tested directly by studying the various contributions of the different ACh cell groups in the BF to both attention and memory functions.

Our results implicate the basal forebrain cholinergic system in attentional and mnemonic processing. Importantly, however, our observations suggest that memory deficits caused by cholinergic depletions may not be directly attributable to an attentional deficit, although aspects of attention (such as target omissions) may be equally compromised. It is more likely that the cortical cholinergic system modulates both attentional and mnemonic processing, perhaps because of its capacity to generally enhance signal-to-noise ratios (Gu 2002). Together, these data have implications for attempts to model cognitive functions produced by cholinergic lesions with relatively selective actions on attention and memory. The simultaneous assessment of attention and its effect on memory in the CAM task provides us with a valuable method for modeling effects of neurochemical manipulations on component cognitive processes in disorders of cognitive function. 
A

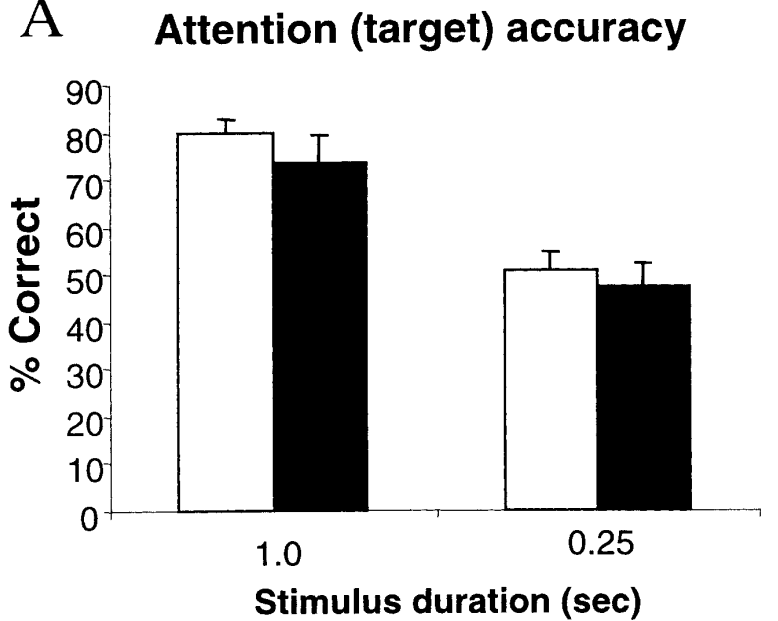

B Memory (choice) accuracy

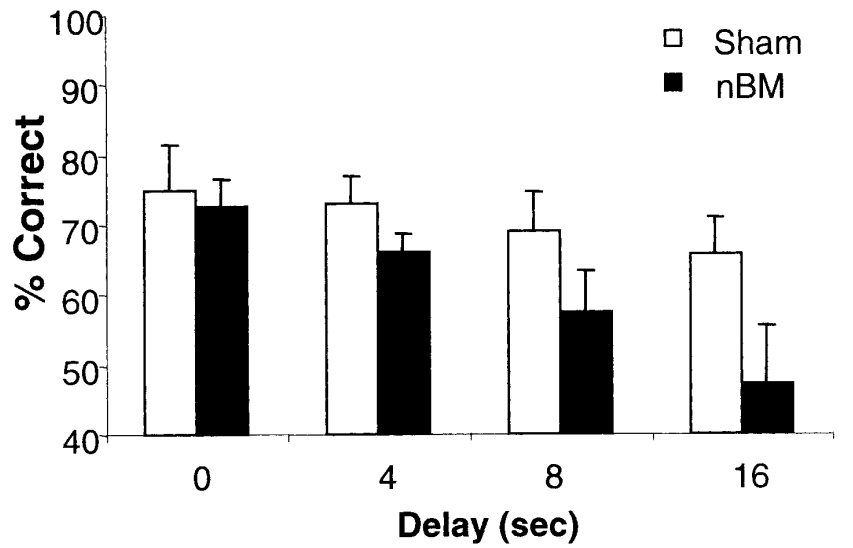

Figure 5 Effects of $n B M$ lesions on the CAM task when the stimulus duration was reduced to 0.25 sec. Mean ( \pm SEM) performance on attentional (target) accuracy $(A)$ and memory (choice) phase $(B)$.

\section{MATERIAL AND METHODS}

\section{Subjects}

All subjects were male Lister Hooded rats (Charles River), housed in pairs in a temperature controlled room $\left(22^{\circ} \mathrm{C}\right)$, under diurnal conditions ( $12 \mathrm{~h}$ light/12 $\mathrm{h}$ dark). Rats were food deprived and maintained at $85 \%$ of their free-feeding weight throughout the experiment. All testing occurred at a regular time during the light period, and animals were 3-months-of-age (230-260 g) at the start of behavioral training. All experimental procedures were subject to UK Home Office approval (Project Licence PPL 80/ 1324).

\section{Apparatus}

Six nine-hole boxes (Cambridge Cognition) were used for the combined attention-memory (CAM) task. This apparatus has been described previously (see Carli et al. 1983) and is used primarily to assess continuous performance in rats (Muir 1996; Passetti et al. 2002). In brief, each box was a $25 \times 25-\mathrm{cm}$ aluminium chamber with an arc of contiguous apertures numbered one to nine (each $2.5 \mathrm{~cm}$ square, $4 \mathrm{~cm}$ deep, and set $2 \mathrm{~cm}$ above floor level). Illumination of each hole was provided by a standard $3 \mathrm{~W}$ bulb located at the rear of the hole. In addition, located at the entrance of each aperture was an infrared photocell beam monitoring the nose poke responding of the rat. Each aperture could be blocked by a metal cover when not required. For the present study, apertures $1,3,5,7$, and 9 were open. Pellets were delivered in a food magazine that was located in the wall opposite the visual array. The opening of the food magazine was covered by a hinged Perspex panel door and was monitored by a microswitch. The four chambers were housed individually within soundattenuating cabinets and were ventilated by low-level noise fans, which also served to mask extraneous background noise. The apparatus and on-line data collection was controlled by means of an Acorn computer system with software written by Dr. R.N. Cardinal (Cambridge University, Cambridge, United Kingdom).

\section{Behavioral Procedure}

Each trial consisted of two phases, the target (attention) phase and the choice (memory) phase. The target phase was similar to the 5-choice serial reaction time task (Carli et al. 1983), although the final requirement for the target duration was less stringent (0.7 sec for experiment 1 and $1.0 \mathrm{sec}$ for experiment 2 ). Rats were trained to discriminate a brief visual stimulus presented randomly in one of the five spatial locations. At the beginning of trial, the house light was illuminated and the trial was initiated by the rat making an entry into the food magazine. After a fixed 5-sec interval, the light at the rear of one of the apertures was illuminated for a short period $(0.7$ or $1.0 \mathrm{sec})$. A nose-poke response in the aperture during illumination and for 5 sec afterward (the limited hold period) was recorded as a correct response. Responses in a nonilluminated hole during the target presentation period (incorrect response) and failures to respond within the limited hold period (omission) terminated the trial. The next trial began following a 10-sec intertrial interval, during which all lights were extinguished.

If the rat made a correct target response, a delay interval was initiated that was signified by the illumination of the food magazine. The animal was required to nose poke the food magazine during the delay. The last nose poke after the delay interval resulted in the choice (memory) phase when two lights were presented simultaneously for $3 \mathrm{sec}$. One choice stimulus light was presented in the hole that was identical to the target light during the attention phase of the trial. The second distractor choice stimulus light was a random light that occurred in one of the remaining four holes. The rat was rewarded with two pellets if it made a nose-poke response in the choice hole that was the same as the original target light (correct choice response) while it was illuminated, and after, for $5 \mathrm{sec}$ (the limited hold period). A nosepoke response in the distractor choice hole or in any of the nonilluminated holes (incorrect choice response) or a failure to respond (choice omission) resulted in the termination of the trial and no food was delivered. The next trial began after an intertrial interval of darkness for $10 \mathrm{sec}$, after which the houselight was illuminated. Each session consisted of 100 trials.

Importantly, the proximity of the choice stimulus lights during the memory phase could be presented at extreme locations. For example, on some trials, the choice lights could be presented in holes 1 and 2, whereas on other trials, the locations could be holes 1 and 5 . Although we cannot dismiss the potential confound that discrimination might be easier when the stimulus lights were further apart from each other than when presented in adjacent holes, a comparison of the accuracy as a function of hole proximity (holes 1 and 2 vs. holes 1 and 5) revealed no significant differences on the last preoperative training day [accuracy: $F(1,12)=0.122, P>0.05$; hole proximity: $F(1$, $12)=0.122, P>0.05]$. In addition, of the nine holes that were available in the apparatus, four were occluded, leaving a space between each hole. Therefore, even when the choice lights were presented in adjacent holes, they were always one hole apart allowing for easier discrimination.

Given the demanding requirements of the CAM task, it took $\sim 4-6$ mo to train animals on this task. Therefore, initial training was conducted according to two phases. The animals were first trained to detect the target; thus, the stimulus duration and lim- 
ited hold period were both set at $1 \mathrm{~min}$. These variables were altered on subsequent sessions according to the animal's performance, until the animal was able to detect a target duration of $0.7-1.0 \mathrm{sec}$. The animal was required to attain $>80 \%$ correct responses accompanied by $<20 \%$ omissions over five consecutive sessions within a 30-min session time. Approximately 25 sessions were required for the animals to attain this criterion.

Following successful training of the target phase, the delay intervals were instituted between the target (attention) and the choice (memory) phases of the task. During initial training, the delay was set at 0 sec for the entire session. The animal was rewarded for making a correct target response and a correct choice response. When animals were able to complete $>70 \%$ correct choice responses at the 0 -sec delay for five consecutive days, the reward pellet following the correct target was removed; thus, animals were only rewarded for making a correct choice response in the memory phase only. Additional delays of 0-16 sec were then introduced according to the animal's performance. When rats were showing reliable performance across five consecutive days with a criterion of $\geq 70$ correct during the target phase and at least 60 correct during the choice phase, the animals were ready for surgery. Approximately 90 sessions were required to attain this criterion.

Following implantation of guide cannulae in the mPFC (experiment 1), the animals were retested on the standard baseline schedule until stable performance was obtained (5 d). Subsequently, these animals were infused with scopolamine prior to testing on the CAM task. In experiment 2, animals with 192 IgG-saporin lesions of the nucleus basalis magnocellularis (nBM) were also retested on the standard baseline schedule. The difficulty of the task was then increased by reducing the stimulus duration in one session to $0.25 \mathrm{sec}$.

\section{Performance Measures}

Several performance measures were recorded for each phase for each trial. For the target (attention) phase, the following measures were calculated:

1. Attentional accuracy was the number of correct target responses/total number of target responses expressed as a percentage. Thus, this measures errors of commission without including errors of omission.

2. Errors of target omission were also recorded for this phase, as the number of trials on which no response was made expressed as a percentage. This measure reflects possible failures in detection.

3. Premature responses were the number of nose-poke responses in the apertures during the interval prior to the onset of the target. This measure reflects possible deficits in impulse control and is related to response preparation.

4. Target-response latency was defined as the time between the onset of the visual target and the point at which the animal made a correct target response.

For the choice (memory) phase, the following measures were calculated:

1. Choice accuracy was the number of correct choice responses/ total number of choice responses expressed as a percentage for each delay.

2. Choice response latency was the time from when the animal made the last nose poke in the food magazine after the delay to when the animal made a correct choice response.

3. Magazine latency was the time between the correct choice response and the time the rat entered the food magazine to collect its food reward.

In addition, the CAM task may well be subject to mediating behaviors similar to the standard delayed nonmatching to position task (Dunnett et al. 1990; Chudasama and Muir 1997), in which behaviors directed at the food magazine appear to facilitate correct responding. In the CAM task, the food magazine is in the opposite direction to the visual display, preventing the animal from using magazine oriented behaviors. Nonetheless, it was still possible that the animals could mediate by nose poking in the same hole as the target stimulus hole in order to facilitate memory. For this reason, we calculated the number of nose-poke responses during the delay that were made in the same hole relative to all other holes. Statistical analyses failed to show any significant differences in these measures throughout the entire testing regimen, suggesting to us that mediating behaviors in the CAM task were certainly minimized, if not perfectly eliminated. Therefore, responses to the same and other holes during the delay were combined to provide a total number of perseverative nose-poke responses during the delay.

\section{Surgery}

\section{Cannulae Implantation}

After training on the CAM task, animals underwent stereotaxic implantation of chronic double-guide cannula. Each guide cannula consisted of a plastic square comprising two 23-guage metal tubes that were $1.5 \mathrm{~mm}$ apart, projecting $3 \mathrm{~mm}$ from the pedestal (Plastics One). Animals were deeply anaesthetised by an intramuscular injection of xylazine (Rompun, Vet Drug; $10 \mathrm{mg} / \mathrm{kg}$ ) and ketamine (Ketaset, Vet Drug; $50 \mathrm{mg} / \mathrm{kg}$ ) and placed in a stereotaxic frame fitted with atraumatic bars (David Kopf Instruments). The incisor bar was set at $-3.3 \mathrm{~mm}$. A small quantity of ophthalmic ointment (Lacri-Lube) was gently wiped over each eye to prevent desiccation of the corneal surfaces. The scalp was retracted to expose the skull, and craniotomies were made directly above the target region of the brain. The guide cannulae was mounted on the arm of the stereotaxic frame and was lowered through the craniotomies at the following coordinates from bregma; AP + 3.0, L +/ - 0.7, DV - 2.2 (from dura). The cannula was then implanted and affixed with dental cement and stainless sterile screws that served to hold the cannula in place. Sterile (dummy) stylets (Plastic One) were introduced in the guide cannula to prevent occlusion. Particular care was taken to minimize infection. Therefore, during a 10-d recovery period, the dummy stylets were changed every other day. Rats were housed individually for the subsequent testing period. A total of 14 rats were cannulated.

\section{Microinfusion Procedure}

Following post operative recovery, rats were initially adapted to two mock infusion protocols to minimize any stress associated with the procedure. Rats were gently restrained while the dummy stylets were removed and replaced with a 30-gauge stainless-steel double injector extending $1.5 \mathrm{~mm}$ beyond the tip of the guide cannula. The double injectors were connected by Portex fine bore polythene tubing (Portex Ltd) to two $10-\mu \mathrm{L}$ precision syringes (SGE) mounted in a Harvard Apparatus infusion pump. Drug or vehicle was infused bilaterally in a volume of $0.5 \mu \mathrm{L}$ over $2 \mathrm{~min}$. The cannulae were left in place for a further $2 \mathrm{~min}$ before behavioral testing.

\section{Drug Preparation}

Scopolamine hydrobromide was dissolved in sterile PBS ( $\mathrm{pH} 7.0$ 7.2) (RBI). All animals received two doses (3 or $10 \mu \mathrm{g})$ or vehicle according to a latin square design. Injections were administered immediately before testing. Each drug-test day was followed by a drug-free day, when no behavioral testing occurred. Animals were then tested on the baseline schedule of the task before the next treatment.

\section{Saporin Lesions of the $n B M$}

Subjects were administered ketamine (Ketaset, Vet Drug; $90 \mathrm{mg} /$ $\mathrm{kg}$, i.p.) in combination with xylazine (Rompun, Vet Drug; 6.7 $\mathrm{mg} / \mathrm{kg}$, i.p.) to induce anaesthesia before being secured in a flat skull position (incisor bar set at $-3.3 \mathrm{~mm}$ relative to the interaural line). A small quantity of ophthalmic ointment (Lacri-Lube) was wiped gently over each eye to prevent desiccation of the corneal surfaces. Lesions of the cortically projecting cholinergic neurons of the nucleus basalis magnocellularis (nBM) were made using the immunotoxin 192 IgG-saporin (Chemicon). Bilateral 
microinfusions of vehicle (PBS, pH 7.4) or 192 IgG-saporin (125 ng/infusion, $0.5 \mu \mathrm{L}$ infusion vol) were carried out using 31-gauge, nonbeveled stainless-steel injectors (Cooperneedle works) at a rate of $0.25 \mu \mathrm{L} / \mathrm{m}$, commencing 2 min after lowering the injectors in the brain. The following stereotaxic coordinates were used (relative to bregma and the dural surface): AP $-0.5 \mathrm{~mm} ; \mathrm{L} \pm 2.9$ $\mathrm{mm}$; V - $7.2 \mathrm{~mm}$ (Paxinos and Watson 1997). Following each infusion, the injector was left in place for 4 min before being slowly retracted. Animals were given a full week to recover from surgery before being retested and housed individually for subsequent testing.

\section{Histological Procedures}

At the conclusion of the behavioral testing, the animals were perfused transcardially with $0.01 \mathrm{M}$ PBS followed by $4 \%$ paraformaldehyde. The cannulae were carefully removed from the brain and discarded. After dehydration by immersion in $20 \%$ sucrose overnight, the brains were sectioned on a freezing microtome. For brains with cannulae implantations, every second section $(60 \mu \mathrm{m})$ was mounted on glass slides and stained with Cresyl Violet. The sections were used to verify cannulae placement.

\section{Neuronal Specificity of $192 \lg G$-Saporin}

An assessment was made of the specificity of the 192 IgG-saporin for cholinergic and parvalbumin (PARV) containing GABAergic neurons of the basal forebrain. Following perfusions and dehydration according to the schedule above, every fourth section (40 $\mu \mathrm{m})$ was stained for choline acetyletransferase (ChAT)-positive cells, and every third section was stained for PARV-positive cells. The histological procedure protocol and histological quantification for these staining techniques was identical to that provided by McGaughy et al. (2002). Cresyl violet staining was used to determine the extent of any general neuronal loss or degeneration in the region of the infusion.

\section{Data Analysis}

Data for each variable were subjected to repeated measures of ANOVA using the SPSS statistical package, version 9.0 (SPSS Inc.). Data were initally explored using boxplots and tests of homogeneity of variance, so that outliers were identified and skewed data, which violate the distribution requirement of the ANOVA were transformed appropriately (arcsine, square root, or logarithmic) as recommended by Winer (1971). Homogeneity of variance across groups was assessed by the Mauchly Sphericity Test. When data sets significantly violated this requirement for a repeated measures design, the Huynh-Feldt $\varepsilon$ was used to calculate a more conservative $P$-value for each F-ratio. The criterion for statistical significance was a probability level of $P<0.05$. For experiment 1 , the within subject factor was dose (three levels vehicle; $3 \mu \mathrm{g}$, and $10 \mu \mathrm{g})$ and the within-subjects factor included session $(5 \mathrm{~d})$ and delay ( 4 levels; $0,4,8$, and $16 \mathrm{sec}$ ). For experiment 2 , the betweensubject factor was lesion [2 levels; Sham controls and nBMsaporin $(\mathrm{nBM})]$, the within-subject factor included session ( $8 \mathrm{~d})$ and delay (4 levels; $2,4,8$, and $16 \mathrm{sec}$ ), and the stimulus duration $(1.0$ and $0.25 \mathrm{sec})$ as appropriate.

\section{ACKNOWLEDGMENTS}

This work was supported by a Programme grant from the Wellcome Trust (TWR) and was completed within the Medical Research Council Cambridge Centre for Behavioural and Clinical Neuroscience. We thank David Theobald for assistance with histological preparation and immunohistochemical staining. F.N. was supported by an MRC studentship, and P.B. was supported by Bayer AG. Y.C. was supported by the Cambridge Cognition.

The publication costs of this article were defrayed in part by payment of page charges. This article must therefore be hereby marked "advertisement" in accordance with 18 USC section 1734 solely to indicate this fact.

\section{REFERENCES}

Barense, M.D., Fox, M.T., and Baxter, M.G. 2002. Aged rats are impaired on an attentional set-shifting task sensitive to medial frontal cortex damage in young rats. Learn. Mem. 9: 191-201.

Bartus, R.T., Dean, R.L., Beer, B., and Lippa, A.S. 1982. The cholinergic hypothesis of geriatric memory dysfunction. Science 207: 408-417.

Bartus, R.T., Flicker, C., Dean, R.L., Pontecorvo, M., Figueirdo, J.C., and Fisher, S.K. 1985. Selective memory loss following nucleus basalis lesions: Long term behavioral recovery despite persistent cholinergic deficiencies. Pharm. Biochem. Behav. 23: 125-135.

Baxter, M.G. and Chiba, A.A. 1999. Cognitive functions of the basal forebrain. Curr. Opin. Neurobiol. 9: 178-183.

Baxter, M.G., Bucci, D.J., Gorman, L.K., Wiley, R.G., and Gallagher, M. 1995. Selective immunotoxin lesions of the basal forebrain cholinergic cells: Effects on learning and memory in rats. Behav. Neurosci. 109: 714-722.

Broks, P., Preston, G.C., Traub, M., Poppleton, P., Ward, C., and Stahl, S.M. 1988. Modelling dementia: Effects of scopolamine of memory and attention. Neuropsychologia 26: 685-700.

Candy, J.M., Perry, R.H., Perry, E.K., Irving, D., Blessed, G., Fiarbairn, A., and Tomlinson, B.E. 1983. Pathological changes in the nucleus of Meynert in Alzhemier's and Parkinson's disease. J. Neurol. Sci. 54: $277-289$.

Carli, M., Robbins, T.W., Evenden, J.L., and Everitt, B.J. 1983. Effects of lesions to ascending noradrenergic neurons on performance on a 5-choice serial reaction time task in rats: Implications for theories of dorsal noradrenergic bundle function based on selective attention and arousal. Behav. Brain Res. 9: 361-380.

Chappell, J., McMahan, R., Chiba, A., and Gallagher, A. 1998. A re-examination of the role of basal forebrain cholinergic neurons in spatial working memory. Neuropharmacology 37: 481-487.

Chiba, A.A., Bucci, D.J., Holland, P.C., and Gallagher, M. 1995. Basal forebrain cholinergic lesions disrupt increments but not decrements in conditioned stimulus processing. J. Neurosci. 15: 7315-7322.

Chiba, A.A., Bushnell, P.J., Oshiro, W.M., and Gallagher, M. 1999. Selective removal of cholinergic neurons in the basal forebrain alters cued target detection in rats. NeuroReport 10: 3119-3123.

Chudasama, Y. and Muir, J.L. 1997. A behavioural analysis of the delayed non-matching to position task: The effects of scopolamine, lesions of the fornix and of the prelimbic region on mediating behaviours by rats. Psychopharmacology 134: 73-82.

Chudasama, Y., Passetti, F., Rhodes, S.E.V., Lopian, D., Desai, A., and Robbins, T.W. 2003. Dissociable aspects of performance on the 5 choice serial reaction time task following lesions of the dorsal anterior cingulate, infralimbic and orbitofrontal cortex in the rat: Differential effects on selectivity, impulsivity and compulsivity. Behav. Brain Res. 146: 105-119.

Colquhoun, W.P. 1962. Effects of hyoscine and meclozine on vigilance and short term memory. Br. J. Indust. Med. 19: 287-296.

Davies, P. and Maloney, A.J.F. 1976. Selective loss of central cholinergic neurons in Alzheimer's disease. Lancet ii: 1403.

Delatour, B. and Gisquet-Verrier, P. 1999. Lesions of the prelimbic-infralimbic cortices in rats do not disrupt response selection processes but induce delay-dependent deficits: Evidence for a role in working memory? Behav. Neurosci. 113: 941-955.

Dias, R. and Aggleton, J.P. 2002. Effects of selective excitotoxic prefrontal lesions on acquisition of nonmatching- and matching-to-place in the T-maze in the rat: Differential involvement of the prelimbic-infralimbic and anterior cingulate cortices in providing behavioural flexibility. Eur. J. Neurosci. 12: 4457-4466.

Drachman, D.A. and Leavitt, J. 1974. Human memory and the cholinergic system. Arch. Neurol. 30: 113-131.

Dunnett, S.B. and Barth, T.M. 1991. Animal models of Alzheimer's disease and dementia (with an emphasis on cortical cholinergic systems. In Behavioral models in psychopharmacology: Theoretical, industrial and clinical perspectives (ed. P. Willner), pp. 359-418. Cambridge University Press, New York.

Dunnett, S.B., Rogers, D.C., and Jones, G.H. 1989. Effects of nucleus basalis magnocellularis lesions in rats on delayed matching and non-matching to position tasks. Eur. J. Neurosci. 1: 395-406.

Dunnett, S.B., Wareham, A.T., and Torres, E.M. 1990. Cholinergic blockade in the prefrontal cortex and hippocampus disrupts short-term memory in rats. NeuroReport 1: 61-64.

Dunnett, S.B., Everitt, B.J., and Robbins, T.W. 1991. The basal forebrain-cortical cholinergic system: Interpreting the functional consequences of excitotoxic lesions. Trends Neurosci. 14: 494-501.

Etherington, R., Mittleman, G., and Robbins, T.W. 1987. Comparative effects of nucleus basalis and fimbria-fornix lesions on delayed matching and alternation tests of memory. Neurosci. Res. Comm. 1: $135-143$.

Everitt, B.J. and Robbins, T.W. 1997. Central cholinergic systems and 
cognition. Annu. Rev. Psychol. 48: 649-684.

Fibiger, H.C. 1991. Cholinergic mechanisms in learning, memory and dementia: A review of recent evidence. Trends Neurosci. 14: 220-223.

Granon, S., Vidal, C., Thinus-Blanc, C., Changeus, J.P., and Poucet, P. 1994. Working memory, response selection and effortful processing in rats with medial prefrontal lesions. Behav. Neurosci. 108: 883-891.

Granon, S., Poucet, B., Thinus-Blanc, C., Changeux, J.P., and Vidal C. 1995. Nicotinic and muscarinic receptors in the rat prefrontal cortex: Differential roles in working memory, response selection and effortful processing. Psychopharmacology 119: 139-144.

$\mathrm{Gu}$, Q. 2002. Neuromodulatory transmitter systems in the cortex and their role in cortical plasticity. Neuroscience 111: 815-835.

Hagan, J.J. and Morris, R.G.M. 1988. The cholinergic hypothesis of memory: A review of animal experiments. In Handbook of psychopharmacology, psychopharmacology of the aging nervous system (eds. L.L. Iversen et al.), Vol. 20, pp. 237-323. Plenum Press, New York.

Himmelheber, A-M., Sarter, M., and Bruno, J.P. 2001. The effects of manipulations of attentional demand on cortical acetylcholine release. Cogn. Brain Res. 12: 353-370.

Jones, D.M., Jones, M.E.L., Lewis, M.J., and Spriggs, T.L.B. 1979. Drugs and human memory: Effects of low doses of nitrazepam and hyoscine on retention. Br. J. Clin. Pharmacol. 7: 479-483.

Kesner, R.P. 2000. Subregional analysis of mnemonic functions of the prefrontal cortex in the rat. Psychobiology 28: 219-228.

Luiten, P.G.M., Gaykema, R.P.A., Traber, J., and Spencer, D.G. 1987. Cortical projection patterns of magnocellular basal nucleus subdivisions as revealed by anterogradely transported phaseolus vulgaris leuocoagglutinin. Brain Res. 413: 229-250.

McGaughy, J., Kaiser, T., and Sarter, M. 1996. Behavioral vigilance following infusions of 192 IgG-saporin into the basal forebrain: Selectivity of the behavioral and relation to cortical AChE-positive fibre density. Behav. Neurosci. 111: 247-265.

McGaughy, J., Dalley, J.W., Morrison, C., Everitt, B.J., and Robbins, T.W. 2002. Selective behavioral and neurochemical effects of cholinergic lesions produced by intrabasalis infusions of 192 IgG-saporin on attentional performance in a five-choice serial reaction time task. J. Neurosci. 22: 1905-1913.

Mesulam, M., Mufson, E.J., Levey, A.I., and Wainer, B.H. 1983a. Central cholinergic pathways in the rat: An overview based on alternative nomenclature (Ch1-Ch6). Neuroscience 10: 1185-1201.

. 1983b. Cholinergic innervation of cortex by the basal forebrain: Cytochemistry and cortical connections of the septal area, diagonal band nuclei, nucleus basalis (substantia innominata and hypothalamus) in the rhesus monkey. J. Comp. Neurol.

214: $170-197$.

Muir, J.L. 1996. Attention and stimulus processing in the rat. Cogn. Brain Res. 3: 215- 225.

Muir, J.L., Everitt, B.J., and Robbins, T.W. 1996. The cerebral cortex of the rat and visual attentional function: Dissociable effects of mediofrontal, cingulate, anterior dorsolateral, and parietal cortex lesions on a five-choice serial reaction time task. Cereb. Cortex 6: $470-481$.

Passetti, F., Chudasama, Y., and Robbins, T.W. 2002. The frontal cortex of the rat and visual attentional performance: Dissociable functions of distinct medial prefrontal subregions. Cereb. Cortex. 12: $1254-1268$.

Paxinos, G. and Watson, C. 1997. The rat brain in stereotaxic coordinates. Academic Press, New York.

Perry, E.K., Perry, R.H., Blessed, G., and Tomlinson, B.E. 1977. Necropsy evidence of central cholinergic deficits in senile dementia. Lancet i: 189 .

Perry, E.K., Tomlinson, B.E., Blessed, G., Bergman, K., Gibson, P.H., and Perry, R.H. 1978. Correlation of cholinergic abnormalities with senile plaques and mental test scores in senile dementia. Brit. Med. J. ii: $1457-1459$.

Petersen, R.C. 1977. Scopolamine induces learning failures in man. Psychopharmacology 52: 283-289.

Ragozzino, M.E., Adams, S., and Kesner, R.P. 1998. Differential involvement of the dorsal anterior cingulate and prelimbic-infralimbic areas of the rodent prefrontal cortex in spatial working memory. Behav. Neurosci. 112: 293-303.

Richardon, R.T. and DeLong, M.R. 1988. A reappraisal of the functions of the nucleus basalis of Meynert. Trends Neurosci. 11: 264-267.

Risbrough, V., Bontempi, B., and Menzaghi, F. 2002. Selective immunolesioning of the basal forebrain cholinergic neurons in rats: Effect on attention using the 5-choice serial reaction time task. Psychopharmacology 164: 71-81.

Robbins, T.W. 2002. The 5-choice serial reaction time task: Behavioural pharmacology and functional neurochemistry. Psychopharmacology 163: $362-380$

\section{Learning \& Memory}

www.learnmem.org
Robbins, T.W., Everitt, B.J., Marston, H.M., Wilkinson, J., Jones, G.H., and Page, K.J. 1989. Comparative effects of ibotenic acid and quisqualic acid-induced lesions of the substantia innominata on attentional function in the rat: Further implications for the role of the cholinergic neurons of the nucleus basalis in cognitive processes. Behav. Brain Res. 35: 221-240.

Robbins, T.W., Semple, J., Kumar, R., Truman, M.I., Shorter, J., Ferraro, A., Fox, B., McKay, G., and Matthews, K. 1997. Effects of scopolamine on delayed-matching-to-sample and paired associates test on visual memory and learning in human subjects: Comparison with diazepam and implications for dementia. Psyhopharmacology 134: $95-106$.

Robbins, T.W., Granon, S., Muir, J.L., Durantou, F., Harrison, A., and Everitt, B.J. 1998. Neural systems underlying arousal and attention. Implications for drug abuse. Ann. NY Acad. Sci. 846: 222-237.

Rossner, S., Schliebs, R., Perez-Polo, J.R., Wiley, R.G., and Bigl, V. 1995. Differential changes in cholinergic markers from selected brain regions after specific immunolesion of the rat cholinergic basal forebrain system. J. Neurosci. 40: 31-43.

Rosvold, H.E., Mirsky, A.F., Sarason, I., Bransome, E.B., and Beck, L.H. 1956. A continuous performance test of brain damage. J. Consult. Psychol. 20: $343-350$.

Sahakian, B.J. 1988. Cholinergic drugs and human cognitive performance. In Handbook of psychopharmacology, psychopharmacology of the aging nervous system (eds. L.L. Iversen et al.), Vol. 20, pp. 393-424. Plenum Press, New York.

Sahakian, B.J., Downes, J.J., Roberts, A.C., Philpot, M., Levy, R., and Robbins, T.W. 1990. Preserved attentional function and impaired mnemonic function in dementia of the Alzheimer type. Neuropsychologia 28: 1197-1213.

Saper, C.B. 1984. Organization of cerebral cortical afferent systems in the rat. I. Magnocellular basal nucleus. J. Comp Neurol. 222: 313-342.

Sarter, M. and Bruno, J.P. 1999. Abnormal regulation of corticopetal cholinergic neurons and impaired information processing in neuropsychiatric disorders. Trends Neurosci. 22: 67-74.

Schliebs, R., Rossner, S., and Bigl, V. 1996. Immunolesion by 192IgG-saporin of rat basal forebrain cholinergic system: A useful tool to produce cortical cholinergic dysfunction. Prog. Brain Res. 109: 253-264

Stoehr, J.D., Mobley, S.L., Roice, D., Brooks, R., Baker, L.M., Wiley, R.G., and Wenk G.L. 1997. The effects of selective cholinergic basal forebrain lesions and aging upon expectancy in the rat. Neurobiol. Learn. Mem. 67: 214-227.

Torres, E.M., Perry, T.A., Blokland, A., Wilkinson, L.S., Wiley, R.G., Lappi, D.A., and Dunnett, S.B. 1994. Behavioural, histochemical and biochemical consequences of selective immunolesions in discrete regions of the basal forebrain cholinergic system. Neuroscience 63: $95-122$.

Turchi, J. and Sarter, M. 1997. Cortical acetylcholine and processing capacity: Effects of cortical cholinergic deafferentation on crossmodal divided attention in rats. Cogn. Brain Res. 6: 147-158.

Waite, J.J., Wardlow, M.L., and Power, A.E. 1999. Deficit in selective and divided attention associated with cholinergic basal forebrain immunotoxin lesion produced by 192 IgG-saporin: Motoric/sensory deficit associated with purkinje cell immunotoxic lesion produced by OX7-192 IgG-saporin. Neurobiol. Learn. Mem. 71: 325-352.

Weingartner, H. 1985. Models of memory dysfunction. Ann. NY Acad. Sci. 444: $359-369$.

Wenk, G.L. 1997. The nucleus basalis magnocellularis cholinergic system: One hundred years of progress. Neurobiol. Learn. Mem. 67: 85-95.

Wenk, G.L., Stoehr, J.D., Quintana, G., Mobley, S., and Wiley, R.G. 1994. Behavioral, biochemical, histological, and electrohysiological effects of $192 \mathrm{IgG}$-saporin injections into the basal forebrain of rats. J. Neurosci. 14: 5986-5995.

Wesnes, K. and Warburton, D.M. 1983. Effects of scopolamine on stimulus sensitivity and response bias in a vigilance task. Neuropsychobiology 9: 154-157.

. 1984. Effects of scopolamine and nicotine on human rapid information processing. Psychopharmacology 82: 147-150.

Whitehouse, P.J., Price, D.L., Struble, R.G., Clark, A.W., Coyle, J.T., and DeLong, M.R. 1982. Alzheimer's disease and senile dementia: Loss of neurons in the basal forebrain. Science 215: 1237-1239.

Winer, B.J. 1971. Statistical principles in experimental design. McGraw-Hill, New York.

Received September 15, 2003; accepted in revised form December 5, 2003. 


\section{Erratum}

Learning \& Memory 11: 78-86 (2004)

Cholinergic Modulation of Visual Attention and Working Memory: Dissociable Effects of Basal Forebrain 192-IgG-saporin Lesions and Intraprefrontal Infusions of Scopolamine Yogita Chudasama, Jeffrey W. Dalley, Falgyni Nathwani, Pascale Bouger, and Trevor W. Robbins

The author F. Nathwani's first name was misspelled and should have been spelled Falguni. 


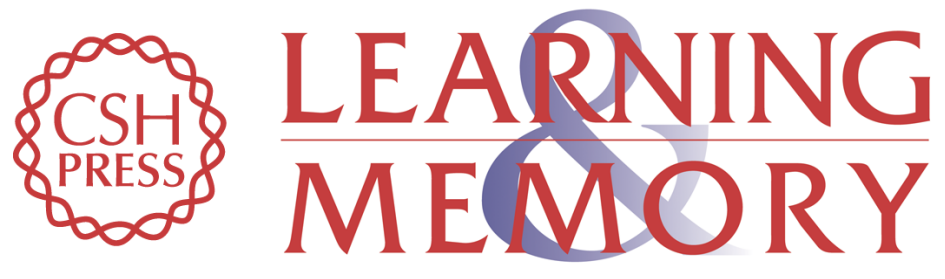

Cholinergic Modulation of Visual Attention and Working Memory: Dissociable Effects of Basal Forebrain 192-IgG-saporin Lesions and Intraprefrontal Infusions of Scopolamine

Yogita Chudasama, Jeffrey W. Dalley, Falgyni Nathwani, et al.

Learn. Mem. 2004, 11:

Access the most recent version at doi:10.1101//m.70904

Related Content Erratum for Volume 11, p. 78

Learn. Mem. March, 2004 11: 227

References This article cites 60 articles, 5 of which can be accessed free at: http://learnmem.cshlp.org/content/11/1/78.full.html\#ref-list-1

Articles cited in:

http://learnmem.cshlp.org/content/11/1/78.full.html\#related-urls

License

Email Alerting Receive free email alerts when new articles cite this article - sign up in the box at the Service top right corner of the article or click here. 